\title{
UCRL-JRNL-224979
}

LAW RENCE LIVERMORE N A T IO N A L LABORATORY

\section{Observations of Plasmons in Warm Dense Matter}

S. H. Glenzer, O. L. Landen, P. Neumayer, R. W. Lee, K. Widmann, S. W. Pollaine, R. J. Wallace, G. Gregori, A. Holl, T. Bornath, R. Thiele, V. Schwarz, W.-D. Kraeft, R. Redmer

October 3, 2006

Physical Review Letters 
This document was prepared as an account of work sponsored by an agency of the United States Government. Neither the United States Government nor the University of California nor any of their employees, makes any warranty, express or implied, or assumes any legal liability or responsibility for the accuracy, completeness, or usefulness of any information, apparatus, product, or process disclosed, or represents that its use would not infringe privately owned rights. Reference herein to any specific commercial product, process, or service by trade name, trademark, manufacturer, or otherwise, does not necessarily constitute or imply its endorsement, recommendation, or favoring by the United States Government or the University of California. The views and opinions of authors expressed herein do not necessarily state or reflect those of the United States Government or the University of California, and shall not be used for advertising or product endorsement purposes. 


\title{
Observations of Plasmons in Warm Dense Matter
}

\author{
S. H. Glenzer, O.L.Landen, P. Neumayer, R. W. Lee, K. Widmann, S. W. Pollaine, and R. J. Wallace \\ L-399, Lawrence Livermore National Laboratory, \\ University of California, P.O. Box 808, Livermore, CA 94551, USA \\ G. Gregori \\ LRC, Rutherford Appleton Laboratory, Chilton, Didcot OX11 OQX, Great Britain Clarendon Laboratory, \\ University of Oxford, Parks Road, Oxford, OX1 3PU, Great Britain.
}

\author{
A. Höll, T. Bornath, R. Thiele, V. Schwarz, W.-D. Kraeft, and R. Redmer \\ Institut für Physik, Universität Rostock, Universitätsplatz 3, D-18051 Rostock, Germany
}

(Dated: September 5, 2006)

\begin{abstract}
We present the first collective x-ray scattering measurements of plasmons in solid-density plasmas. The forward scattering spectra of a laser-produced narrow-band x-ray line from isochorically heated beryllium show that the plasmon frequency is a sensitive measure of the electron density. Dynamic structure calculations that include collisions and detailed balance match the measured plasmon spectrum indicating that this technique will enable new applications to determine the equation of state and compressibility of dense matter.
\end{abstract}

PACS numbers: 52.25.Os, 52.35.Fp, 52.50.Jm

Keywords: Plasmons, Dispersion, Compton scattering, dense Plasmas

Accurate measurements of the physical properties of dense matter including temperature, density, and ionization state are important for understanding and modeling high-energy density science experiments $[1,2]$. Therefore, new and precise techniques [3-7] that make use of $\mathrm{x}$ rays to penetrate dense or compressed materials are broadly applicable in the dense matter community, e.g., [8, 10-17]. Recently, spectrally-resolved xray scattering has been demonstrated in dense plasmas [18] allowing accurate measurements of the electron velocity distribution function, temperature, and ionization state. These scattering experiments have been performed in backscatter geometry, where the scattering process is non-collective and the spectrum shows the Compton down-shifted line that is broadened by the thermal motion of the electrons, thus providing the temperature with high accuracy.

In addition to the Compton scattering feature from inelastic scattering by free and weakly bound electrons, the non-collective scattering spectrum exhibit the unshifted Rayleigh scattering component from elastic scattering by tightly bound electrons. The latter occupy quantum states deep in the Fermi sea that can not be excited due to the Pauli exclusion principle. These electrons become available for inelastic scattering by $\mathrm{x}$ rays and contribute to the Compton feature by excitation and ionization. The intensity ratio of the inelastic Compton to the elastic Rayleigh scattering component can hence be a sensitive measure of the ionization state $[18,19]$. In isochorically heated matter where the ion density is known a priori, the ionization state also provides a measurement of the electron density. However, in many high-energy density physics experiments, definite measurements of material properties such as the equation of state and the optical response, i.e., the reflectivity, transmission, and conductivity, require investigations of dynamically compressed matter or shocked materials. In these conditions, a direct accurate measurement of the electron density has not yet been demonstrated.

In this letter, we present the first observations of plasmons in the warm dense matter regime. The measurements have been performed in well-characterized solid density beryllium targets that have been heated isochorically with a broad-band $\mathrm{x}$ ray source into a weakly degenerate strongly coupled (nonideal) dense plasma state. In these conditions, forward scattering of the narrow-band Ly- $\alpha$ x-ray line at $2.96 \mathrm{keV}$ accesses the collective scattering regime and measures the characteristic plasmon peak associated with the collective plasma (Langmuir) oscillations [20]. The measured spectra are fit with a theoretical form factor $S(\mathbf{k}, \omega)$ that includes collisional damping of plasmons [21, 22] with a Mermin ansatz [6, 7] thus accounting for weak degeneracy effects. The experiment shows that the frequency of the plasmon resonance that is down-shifted from the incident probe radiation by $\Delta E_{P l} \simeq 28 \mathrm{eV}$ is an accurate measurement of the electron density. The experimental results for the density are consistent with radiation-hydrodynamics calculations and with densities inferred from the ionization balance determined by earlier backscatter measurements.

The experiments have been performed employing 27 laser beams of the Omega laser facility [23]. Figure 1 shows a schematic of a scattering target consisting of a $600 \mu \mathrm{m}$ diameter $300 \mu \mathrm{m}$ long solid beryllium cylinder, a $1 \mathrm{~mm}$-long hollow beryllium cylindrical extension, and 4 mm-long Au shields attached on the sides. The central 


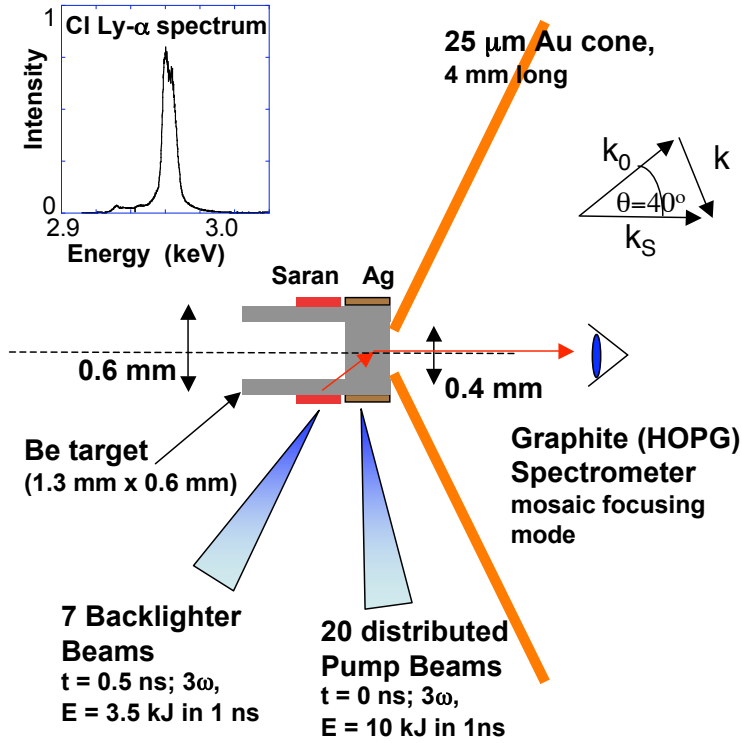

FIG. 1: Experimental setup for collective x-ray forward scattering is shown along with the $\mathbf{k}$-vector diagram and the x-ray probe spectrum .

beryllium cylinder is coated with $1 \mu \mathrm{m}$ thick silver, which we directly illuminate from all sides with twenty 351nm pump laser beams with a total laser energy of $E_{L}=$ $10 \mathrm{~kJ}$ in a 1-ns long square pulse producing $3-4 \mathrm{keV} \mathrm{Ag}$ L-shell radiation. Radiation-hydrodynamic simulations with the code LASNEX [24] indicate that these $\mathrm{x}$ rays heat the bulk beryllium isochorically and homogeneously to electron temperatures of order of the Fermi energy. The calculations show that at the end of the 1 ns-long heating pulse a weakly degenerated solid-density plasma with electron densities of $n_{e}=(2-3) \times 10^{23} \mathrm{~cm}^{-3}$ and temperatures of $T_{e}=(10-15) \mathrm{eV}$ is produced across the inner $400-\mu \mathrm{m}$ diameter of the Be cylinder.

Under these conditions, the collective scattering regime for plasmon measurements has been accessed by forward scattering of the chlorine Ly $-\alpha$ line at the $\mathrm{x}$-ray energy $E_{0}=2.96 \mathrm{keV}$. The $\mathrm{Ly}-\alpha$ line has been produced by illuminating a $12.5 \mu$ m-thick saran $\left(\mathrm{C}_{7} \mathrm{H}_{8} \mathrm{~N} \mathrm{Cl}_{3}\right)$ foil with 7 delayed backlighter beams with a total energy of $3.5 \mathrm{keV}$. A long hollow cylinder was chosen for mounting the saran foil to achieve close proximity of the $\mathrm{x}$ ray backlighter source to the beryllium plasma under investigation; a solid angle of $d \Omega \approx 8 \times 10^{-2}$ has been achieved. Using the empirical conversion efficiency of $\eta=E_{L y-\alpha} / E_{\text {Laser }}=3 \times 10^{-3}$ from Ref. [25] and by taking into account the solid angle, detector integration time, and transmission through the cylinder walls we estimate $10^{14} \mathrm{Cl}$ Ly- $\alpha$ x-ray probe photons at the sample. Together with the cross section for collective scattering and scattering length of $l=400 \mu \mathrm{m}$ we obtain a scattering fraction of $\sigma n_{e} l=2 \times 10^{-3}$ and $2 \times 10^{11}$ scattered photons for single-shot scattering experiments.
The scattered $\mathrm{x}$ rays have been collected through a hole in the center of the Au shields with a gated crystal spectrometer. This geometry results in forward scattering at an average scattering angle of $\theta=40^{\circ}$. A $400 \mu \mathrm{m}$ diameter hole was chosen to restrict the detector view to the central homogeneously heated beryllium plasma. The shields prevent detection of the direct chlorine emission from the foil and of photons scattered by shock waves that compressed the outer $\sim 100 \mu \mathrm{m}$ beryllium by the end of the 1-ns heating pulse.

A graphite (HOPG) crystal in the mosaic focusing mode [18] has been applied to disperse the scattered radiation onto a gated microchannelplate detector with a temporal resolution of $\sim 100$ ps. The HOPG crystal provides high reflectivity [26] allowing temporally-resolved measurements of the scattering spectra with a total detection efficiency of $10^{-7}$ providing $2 \times 10^{4}$ detected photons in a single shot. Further, the spectral resolution of the graphite crystal is 500 . Combined with the narrow spectral bandwidth of the $\mathrm{Cl} \mathrm{Ly}-\alpha$ line that shows negligible dielectronic satellite contributions [cf. Fig. (1)] we achieve an effective spectral resolution of $7 \mathrm{eV}$. This is sufficient to spectrally resolve the plasmons whose spectral shift slightly exceeds the plasma frequency of $\omega_{p}=\left(n_{e} e^{2} / \epsilon_{0} m_{e}\right)^{1 / 2} \simeq 20 \mathrm{eV}$ for our conditions.

For conditions where the frequency of the scattered radiation is close to the incident radiation frequency, i.e., for small momentum transfers, the scattering vector $\mathbf{k}$ is approximated by the relation

$$
k=|\mathbf{k}|=4 \pi \frac{E_{0}}{h c} \sin (\theta / 2) .
$$

In this experiment, we have focused the backlighter beams onto the saran foil in one spot with an effective diameter of $\sim 80 \mu \mathrm{m}$ achieving spatial coherence [5] for the range of scattering angles of $25^{\circ}<\theta<55^{\circ}$ predominantly probing $\mathbf{k}$-vectors with $k=10^{10} \mathrm{~m}^{-1}$. This scattering geometry, the $\mathrm{Cl} \mathrm{Ly}-\alpha$ x-ray probe energy, and the plasma parameters result in collective x-ray scattering (e.g. Refs. [12]) from fluctuations characterized by wavenumbers, $k$ with

$$
\alpha=\frac{1}{k \lambda_{S}}>1 .
$$

Here, $\lambda_{S}$ is the screening length in the plasma that will approach the Debye length for a classical plasma and the Thomas-Fermi length for full degeneracy. In our conditions of a weakly degenerate dense plasma, we calculate the screening length at an effective temperature that correctly includes the high density (degenerate) and low density (classical) limits [5] resulting in $\alpha=1.6$. In this regime, the scattered light spectrum shows collective effects corresponding to scattering resonances off ion acoustic waves and off electron plasma waves, i.e. plasmons.

Figure 2 shows the measured scattering spectrum at $\mathrm{t}=0.7$ ns. Also shown are synthetic scattering profiles 

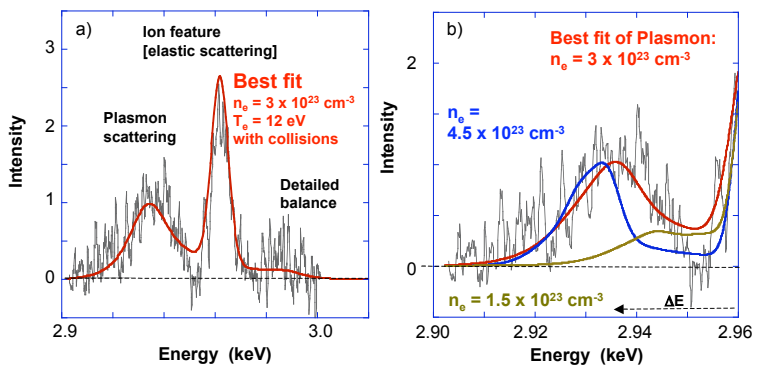

FIG. 2: (a) Experimental scattering data are shown along with the theoretical fit. (b) Comparison of the plasmon spectrum with calculations for three different densities indicating that the spectrum is best fit for $n_{e}=3 \times 10^{23} \mathrm{~cm}^{-3}$.

that represent a convolution of the theoretical form factor $S(\mathbf{k}, \omega)[5-7,18,27]$, calculated for the range of $\mathbf{k}$ vectors of the experiment, with the spectral resolution of $7 \mathrm{eV}$. In Fig. 2(a) the ion feature is observed as an elastic scattering peak at $E_{0}$ that is not resolved in this experiment; the spectral width is determined by the experimental resolution and the intensity is sensitive to the frequency-integrated structure factors $S_{e i}^{2}(\mathbf{k}) / S_{i i}(\mathbf{k})$. On the lower-energy wing of the ion feature, down-shifted in energy from $E_{0}$ by $\Delta E_{p l} \simeq 28 \mathrm{eV}$ we observe a strong plasmon resonance. On the higher-energy wing of $E_{0}$ with the same frequency shift, the data show a weak up-shifted plasmon signal. Compared to the intensity of down-shifted plasmon, the intensity is reduced by the Bose function $e^{\hbar \Delta \omega / k_{B} T}$ reflecting the principle of detail balance. The intensity ratio of these plasmon features is thus sensitive to the temperature. In the present experiment, the signal to noise ratio only allows us to deduce an upper limit of $T_{e}<25 \mathrm{eV}$. We therefore concentrate on the spectrum of the red-shifted plasmon data to provide accurate characterization of the plasma conditions.

The theoretical form factor $S(\mathbf{k}, \omega)$ represents the frequency shift, $\omega_{p l}$, and width, $\gamma$, of the plasmon as determined by the plasmon dispersion relation and by damping processes, respectively. The former can be approximated for small values of $\mathbf{k}$, i.e. large values of $\alpha$, by a modified Bohm-Gross relation

$$
\omega_{p l}^{2}=\omega_{p}^{2}+3 k^{2} v_{t h}^{2}\left(1+0.088 n_{e} \Lambda_{e}^{3}\right)+\left(\frac{\hbar^{2} k^{2}}{2 m_{e}}\right)^{2}
$$

with the thermal velocity, $v_{t h}=\sqrt{k_{B} T / m_{e}}$ and the thermal wave length $\Lambda_{e}=h / \sqrt{2 \pi m_{e} k_{B} T}$. In Eq. (3), the first term represents the well-known contribution from electron oscillations [20], the second term takes into account the propagation of the oscillation due to the thermal pressure [28]. The third term includes the Fermi pressure, thus taking into account degeneracy effects [29], and the last term is the Compton shift.

The Compton shift accounts for the fact that during the scattering process, the incident photon transfers the

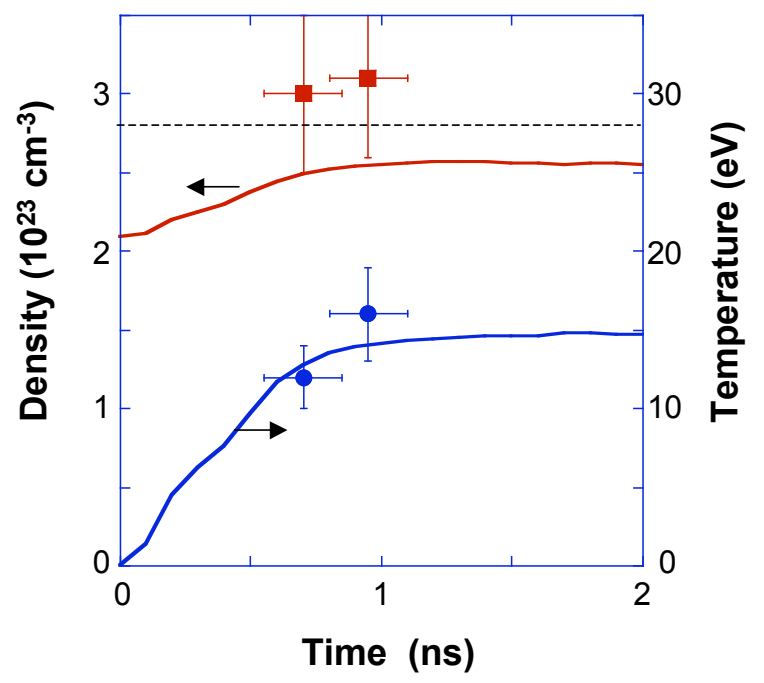

FIG. 3: (a) Plasma parameters from collective x-ray scattering and radiation hydrodynamics calculations are shown. The dashed curve shows the electron density for solid-density beryllium for $Z=2.2$ measured previously in backscattering.

momentum $\hbar \mathbf{k}$ and the energy $\hbar \omega=(\hbar \mathbf{k})^{2} / 2 m_{e}$ to the electron. This quantity depends only on the scattering geometry and the x-ray probe energy resulting in a constant Compton shift of $\Delta E_{C}=4 \mathrm{eV}$ for our conditions. The Compton shift combined with the electron oscillations accounts for most of the measured shift. Therefore, the plasmon spectrum provides a sensitive measure of the electron density leaving the temperature sensitive term as a small correction of the order of the Compton energy.

Figure 2(b) shows the sensitivity of the plasmon spectrum to the electron density. These calculations use the full theoretical form factor taking into account damping processes, the Bose function, and the range of finite $\mathbf{k}$ vector values. The best fit of the experimental data is obtained with the parameters: $n_{e}=3 \times 10^{23} \mathrm{~cm}^{-3}$ and $T_{e}=$ $12 \mathrm{eV}$ when including electron-ion collisions in $S(\mathbf{k}, \omega)$ with a collision frequency of $\nu_{e i}=0.2 \mathrm{Ry}=5 \times 10^{14} \mathrm{~s}^{-1}$. The latter is calculated with first order perturbation theory, i.e., the Born approximation, and included into $S(\mathbf{k}, \omega)$ with a Mermin ansatz $[6,7]$. The theoretical profile for the electron density of $n_{e}=4.5 \times 10^{23} \mathrm{~cm}^{-3}$ yields a plasmon spectrum that overestimates the frequency shift not accounting for the full width of the measured plasmon. On the other hand, a density of $n_{e}=1.5 \times 10^{23} \mathrm{~cm}^{-3}$ results in a spectrum without a resonance feature not consistent with experiment. This comparison shows that the data yield the local density with high accuracy; an error bar of $20 \%$ is obtained in the present experiment.

Figure 3 shows the density and temperature inferred from the plasmon frequency shift and width, repectively. Also shown are the results from radiation-hydrodynamics simulations [24] along with the density inferred from the 
measured ionization balance assuming isochoric heating [18]. In the latter case, an ionization state of $Z=2.2$ has been measured resulting in $n_{e}=(Z / A) \rho 6 \times 10^{23} \mathrm{~cm}^{-3}=$ $2.8 \times 10^{23} \mathrm{~cm}^{-3}$ with mass density $\rho=1.85 \mathrm{~g} / \mathrm{cm}^{-3}$ and atomic number of $A=9$ for Be. This value is close to $Z=2$ delocalized electrons per ion expected for cold beryllium. We find excellent agreement between the Plasmon data and the density inferred from the ionization state while the radiation-hydrodynamics calculations only marginally agree with the data due to approximations in the calculation of $Z$ [18]. In general, determining the electron density from the frequency shift of the plasmon is a robust diagnostics procedure; she shift determined with the Mermin approach agrees with results obtained with the random phase approximation [5] or with static local field corrections [9] to better than $1 \%$.

Besides information on plasmon frequency and electron density, we find that the plasmons are damped by electron-ion collisions. Dynamic form factor calculations, which include collision effects as well as detailed balance fit the plasmon and the ion feature spectra for electron temperatures calculated by the hydrodynamic simulations. Random phase approximation calculations that do not take into account collisions result into plasmon widths that are too narrow. Moreover, the intensity of the ion feature is sensitive to the ion-ion and electron-ion structure factors and consequently to the electron and ion temperatures. For the fit shown in Fig. 2, the relative intensities of the peaks are reproduced with calculations using quantum potentials and with $T_{e}=T_{i}=12 \mathrm{eV}$.

Generally, inferring the temperature from the damping of the plasmons relies on calculations of the collision frequency and is therefore dependent on the specific theoretical approximation [7]. As there are no independent tests of the various models [30] presently available, this experimental method will provide such a test by determining independently the electron temperature simultaneously with plasmon measurements.

In summary, we have demonstrated a novel x-ray scattering technique on plasmons that allows accurate measurements of plasma conditions and the optical properties in warm dense matter. We find that the theoretical form factor fit the measured plasmon spectra, in particular the frequency shift and the spectral width when including collisions. The plasma conditions inferred from the scattering data are in agreement with independent ionization balance measurements and hydrodynamic simulations.

This work was performed under the auspices of the U.S. Department of Energy by the University of California Lawrence Livermore National Laboratory under contract number No. W-7405-ENG-48. This work was supported by LDRD grant No. 05-ERI-003, the VH-VI104 of the Helmholtz Society, the Sonderforschungsbereich SFB 652, and the Alexander-von-Humboldt foundation.

[1] R. Davidson et al., Frontiers in High Energy Density Physics: The X-Games of Contemporary Science (The National Academies Press, Washington, DC, 2003), M. Turner et al., Connecting Quarks with the Cosmos: Eleven Science Questions for the New Century (The National Academies Press, Washington, DC, 2003).

[2] J.D. Lindl, Phys. Plasmas 2, 3933 (1995). J.D. Lindl et al., Phys. Plasmas 11, 339 (2004).

[3] D. Riley et al., Phys. Rev. Lett. 84, 1704 (2000).

[4] O. L. Landen et al., J. Quant. Spectrosc. Radiat. Transfer, 71, 465 (2001).

[5] G. Gregori et al., Phys. Rev. E 67, 026412 (2003), ibid, Europhys. Lett. 7, 1234 (2006).

[6] A. Hoell et al., Eur. Phys. J. D 29159 (2004).

[7] R. Redmer et al., IEEE Trans. Plasma Science 33 , 77 (2005).

[8] S. L. Johnson et al., Phys. Rev. Lett. 91, 157403 (2003), ibid 94, 057407 (2005).

[9] S. Ichimaru Statistical Plasma Physics, Vol. 2 (Addison, New York, 1994).

[10] S.V. Adamjan et al., Europhys. Lett. 25, 11 (1994).

[11] U. Bergmann et al., Phys. Rev. B 66092107 (2002).

[12] W. Schülke, Handbook on Synchrotron Radiation, (Vol. 3, G. Brown, D.E. Moncton (eds.), Elsevier (1991)

[13] Ph. Wernet, Science 304, 995 (2004).

[14] N. Itoh et al., Phys. Rev. Lett. 49, 1932 (1982).

[15] A. Ng et al., Phys. Rev. Lett. 72, 3351 (1994).

[16] R. L. Liboff, J. Appl. Phys. 562530 (1984).

[17] T. R. Boehly et al., Phys. Rev. Lett. 87, 145003 (2001).

[18] S. H. Glenzer et al., Phys. Rev. Lett. 90, 175002 (2003), ibid Phys. Plasmas 10, 2433 (2003).

[19] G. Gregori et al., Phys. Plasmas 11, 2754 (2004). ibid, J. Quant. Spectrosc. Radiat. Transfer, 99, 225 (2006).

[20] L. Tonks and I. Langmuir, Phys. Rev. 33, 195 (1929).

[21] A. N. Mostovych and A. W. DeSilva, Phys. Rev. Lett. 53, 1563 (1984).

[22] O. L. Landen and R. J. Winfield, Phys. Rev. Lett.54, 1660 (1985).

[23] J. M. Soures et al., Fusion Technology, 30, 492 (1996).

[24] G. Zimmerman and W. Kruer, Comments Plasma Phys. Controlled Fusion 2, 85 (1975).

[25] M.K. Urry et al., J. Quan. Spectr. Trans. 99, 636 (2006).

[26] F. J. Marshall and J. A. Oertel, Rev. Sci. Instrum. 68, 735 (1997).

[27] J. Chihara, J. Phys.: Condens. Matter, 12, 231 (2000).

[28] D. Bohm and E. P. Gross, Phys. Rev. 75, 1851 (1949).

[29] R. Zimmerman, Many-Particle Theory of Highly Excited Semiconductors (Teubner, Leipzig, 1987).

[30] H. Reinholz et al., Phys. Rev. E 62, 5648 (2000). 